\title{
AN INVERSE LIMIT REPRESENTATION FOR COMPLETE BOOLEAN ALGEBRAS
}

\author{
by R. BEAZER
}

(Received 7 December, 1970)

1. Introduction. There are two well-known methods to build up algebras from given algebras, the direct and inverse limits, and a systematic account of these constructions may be found in [2]. It is known that every algebra can be represented as a direct limit of finitely generated algebras although in some cases the representation is trivial. Furthermore, Haimo [3] has established a certain inverse limit representation for the class of all infinite Boolean algebras which generalises, in actual fact, to the class of all infinite lattices with 1 . The purpose of this note is to exhibit a certain nontrivial inverse limit representation which is peculiar to the class of infinite, complete Boolean algebras.

2. Preliminaries. The definitions and results of this section are selected from [1] and [2]. An $u p($ down)-directed set is a partially ordered set $\langle I ; \leqq\rangle$ in which every pair of elements has an upper (lower) bound. A nonempty subset $J$ of a partially ordered set $I$ is said to be cofinal in $I$ whenever $\forall \alpha \in I, \exists \beta \in J$ satisfying $\alpha \leqq \beta$. Any cofinal subset of an up-directed set is necessarily up-directed. An inverse family $\mathscr{I}$ of Boolean algebras is defined to be a triplet of the following objects:

(i) An up-directed set $\langle I$; $\leqq$.

(ii) Boolean algebras $\mathscr{B}_{\alpha}=\left\langle\mathscr{B}_{\alpha} ; \cup, \cap,{ }^{\prime}\right\rangle$ for each $\alpha \in I$.

(iii) Homomorphisms $\phi_{\beta \alpha}: \mathscr{B}_{\alpha} \rightarrow \mathscr{B}_{\beta}$ for all $\beta \leqq \alpha(\alpha, \beta \in I)$, such that $\gamma \leqq \beta \leqq \alpha \Rightarrow \phi_{\gamma \alpha}=$ $\phi_{\gamma \beta} \circ \phi_{\beta \alpha}, \phi_{\alpha \alpha}$ is the identity on $\mathscr{B}_{\alpha}$ and, if $1_{\beta}, 0_{\beta}\left(1_{\alpha}, 0_{\alpha}\right)$ are the greatest and least elements of $\mathscr{B}_{\beta}\left(\mathscr{B}_{\alpha}\right)$, then $\phi_{\beta \alpha}\left(1_{\alpha}\right)=1_{\beta}$ and $\phi_{\beta \alpha}\left(0_{\alpha}\right)=0_{\beta}$.

The inverse limit of an inverse family $\mathscr{I}=\left\{\mathscr{B}_{\alpha} ; \phi_{\beta \alpha} \mid \alpha \in I\right\}$ is defined as that subset of the direct product $\prod_{\alpha \in I} \mathscr{B}_{\alpha}$ consisting of all functions $t$ satisfying $\forall(\beta \leqq \alpha), \phi_{\beta \alpha}(t(\alpha))=t(\beta)$; it is a subalgebra of $\prod_{\alpha \in I} \mathscr{B}_{\alpha}$ and is denoted by $\lim _{-}\left\{\mathscr{B}_{\alpha} ; \phi_{\beta \alpha} \mid \alpha \in I\right\}$. If $J$ is any cofinal subset in the index set $I$ of an inverse family $\mathscr{I}$, then the corresponding inverse limits are isomorphic.

If $\mathscr{B}^{\infty}$ denotes the inverse limit of an inverse family $\mathscr{I}=\left\{\mathscr{B}_{\alpha} ; \phi_{\beta \alpha} \mid \alpha \in I\right\}$ of Boolean algebras, then the maps $p_{\alpha}: \mathscr{B}^{\infty} \rightarrow \mathscr{B}_{\alpha}(\alpha \in I)$ defined by $p_{\alpha}(t)=t(\alpha) \forall t \in \mathscr{B}^{\infty}$ are homomorphisms and will be referred to as canonical homomorphisms. If $\phi: \mathscr{B} \rightarrow \mathscr{B}^{\infty}$ is an inverse limit representation of a Boolean algebra $\mathscr{B}$, then the homomorphisms $\phi_{\alpha}=p_{\alpha} \circ \phi: \mathscr{B} \rightarrow \mathscr{B}_{\alpha}$ will be referred to as decomposition homomorphisms. If in any such representation we replace each $\mathscr{B}_{\alpha}$ by its associated subalgebra $\mathscr{B}_{\alpha}^{*}=\left\{t(\alpha): t \in \mathscr{B}^{\infty}\right\}$ and each homomorphism $\phi_{\beta \alpha}: \mathscr{B}_{\alpha} \rightarrow \mathscr{B}_{\beta}$ by its restriction $\phi_{\beta \alpha}^{*}=\phi_{\beta \alpha} \mid \mathscr{B}_{\alpha}^{*}$, then it is evident that $\mathscr{B} \cong \lim _{\leftarrow}\left\{\mathscr{B}_{\alpha}^{*} ; \phi_{\beta \alpha} \mid \alpha \in I\right\}$

is an inverse limit representation of $\mathscr{B}$ in which each decomposition homomorphism and each homomorphism $\phi_{\beta \alpha}^{*}$ is surjective. We shall assume that this replacement has been made in any inverse limit representation. 
Every Boolean algebra $\mathscr{B}$ has, of course, a trivial inverse limit representation, namely, as the inverse limit of the family $\left\{\mathscr{B}_{\alpha} ; \phi_{\beta \alpha} \mid \alpha \in I\right\}$, where $I$ is an arbitrary up-directed set, $\mathscr{B}_{\alpha}=\mathscr{B} \forall \alpha \in I$ and each homomorphism $\phi_{\beta \alpha}$ is the identity on $\mathscr{B}$. It is clear that in any trivial representation all decomposition homomorphisms are bijective. Furthermore, if $\left\{\mathscr{B}_{\alpha} ; \phi_{\beta \alpha} \mid \alpha \in I\right\}$ is an inverse family in which $I$ has a greatest element $\alpha_{1}$, then the singleton subset $\left\{\alpha_{1}\right\}$ is cofinal in $I$ and so $\lim _{\leftarrow}\left\{\mathscr{B}_{\alpha} ; \phi_{\beta \alpha} \mid \alpha \in I\right\} \cong \mathscr{B}_{\alpha_{1}}$. With these remarks in mind we make the following definition.

A Boolean algebra is inversely reducible if and only if it has a representation as an inverse limit of Boolean algebras in which no decomposition homomorphism is an isomorphism.

3. In preparation for the main theorem we recall that a filter $\nabla$ in a Boolean algebra $\mathscr{B}$ is said to be dense whenever it satisfies $\nabla^{* *}=\mathscr{B}$ (where $\nabla^{*}$ denotes the pseudo-complement of $\nabla$ within the lattice of filters of $\mathscr{B}$ ), or, equivalently, whenever its "lower section" $\nabla^{-}=\{a \in \mathscr{B}: a \leqq x \forall x \in \nabla\}$ contains only the element 0 . Finally we need the following

Leмma. In any infinite Boolean algebra there exists at least one proper, dense filter.

Proof. Suppose to the contrary that no proper filter in $\mathscr{B}$ is dense; then in particular no maximal filter in $\mathscr{B}$ can be dense. Let $\nabla$ be an arbitrary maximal filter in $\mathscr{B}$ and suppose that $a \in \nabla^{-}$, so that $a \leqq f \forall f \in \nabla$. Then $a \in \nabla$; for otherwise the join $\nabla_{a}=\nabla \cup(a) \uparrow$ of $\nabla$ and the principal filter $(a) \uparrow=\{x \in \mathscr{B} ; x \geqq a\}$ generated by $a$ properly contains $\nabla$, and maximality yields $\nabla_{a}=\mathscr{B}$. Hence $0=f \cap x$ for some $f \in \nabla$ and $x \geqq a$, which implies that $0=f \cap a=a$ and therefore $\nabla^{-}=\{0\}$, so that $\nabla$ is dense. It follows now that every maximal filter in $\mathscr{B}$ is principal or, equivalently, that $\mathscr{B}$ is finite, contrary to the hypothesis of the lemma.

THEOREM. Any infinite, complete Boolean algebra is inversely reducible.

Proof. If $\mathscr{B}$ is an infinite complete Boolean algebra, then the preceding lemma asserts the existence of a proper, dense filter $\nabla=\left\{a_{\alpha}: \alpha \in I\right\}$. For each $\alpha \in I$, let $\left(a_{\alpha}\right) \downarrow$ denote the principal ideal in $\mathscr{B}$ generated by the element $a_{\alpha}$ of $\nabla$; then, since $\nabla$ is proper, $a_{\alpha} \neq 0 \forall \alpha \in I$ and so $\left(a_{\alpha}\right) \downarrow \neq\{0\}, \forall \alpha \in I$. Furthermore, since $\bigcap_{\alpha \in I} a_{\alpha}$ exists and $\bigcap_{\alpha \in I} a_{\alpha} \leqq a_{\alpha} \forall \alpha \in I$, it follows that $\bigcap_{\alpha \in I} a_{\alpha} \in \nabla^{-}$and so $\bigcap_{\alpha \in I} a_{\alpha}=0$, which implies that $\bigcap_{\alpha \in I}\left(a_{\alpha}\right) \downarrow=\left(\bigcap_{\alpha \in I}^{\alpha \in I} a_{\alpha}\right) \downarrow=\{0\}$. Now, since $\nabla$ is a filter in $\mathscr{B}$, the elements of $\nabla$ are trivially down-directed by the partial ordering inherited from $\mathscr{B}$ and so, if we partially order the index set $I$ by requiring that $\beta \leqq \alpha \leftrightarrow a_{\alpha} \leqq a_{\beta}$, it follows that the pair $\langle I ; \leqq\rangle$ is an up-directed set indexing the family of congruences $\Phi_{\alpha}$ induced by the ideals $\left(a_{\alpha}\right) \downarrow$ of $\mathscr{B}$ in such a way that $\bigcap_{\alpha \in I} \Phi_{\alpha}=\omega$ (the equality relation) and $\beta \leqq \alpha \rightarrow \Phi_{\alpha} \subseteq \Phi_{\beta}$. It follows now that the system of quotient algebras $\mathscr{B}_{\alpha}=\mathscr{B} / \Phi_{\alpha}$ and homomorphisms $\phi_{\beta \alpha}: \mathscr{B}_{\alpha} \rightarrow \mathscr{B}_{\beta}$ defined by $\phi_{\beta \alpha}\left([x]_{\alpha}\right)=[x]_{\beta} \forall x \in \mathscr{B}$ (where $[x]_{\alpha}$ denotes the congruence class modulo $\Phi_{\alpha}$ containing $x$ ) form an inverse family and that, by the classical Birkhoff subdirect product theorem, the correspondence $\phi: \mathscr{B} \rightarrow \mathscr{B}^{\infty}=\lim _{\leftarrow}\left\{\mathscr{B}_{\alpha} ; \phi_{\beta \alpha} \mid \alpha \in I\right\}$ defined by $\phi(x)=t_{x} \in \mathscr{B}^{\infty}$, where $t_{x}(\alpha)=[x]_{\alpha} \forall \alpha \in I$, is an isomorphism from $\mathscr{B}$ onto the subalgebra $\left\{t_{x}: x \in \mathscr{B}\right\}$ of $\mathscr{B}^{\infty}$. Furthermore, since $\Phi_{\alpha} \neq \omega \forall \alpha \in I$, this representation is such that no 
decomposition homomorphism is an isomorphism. To show that $\mathscr{B}$ is inversely reducible it suffices to show that $\phi$ is onto $\mathscr{B}^{\infty}$ and to do this we use the completeness of $\mathscr{B O}$. Let $t$ be an arbitrary element in $\mathscr{B}^{\infty}$ and write $t(\alpha)=\left[x_{\alpha}\right]_{\alpha} \forall \alpha \in I$; then in showing that $\phi$ is surjective, it suffices to show that there exists $x \in \mathscr{B}$ such that $[x]_{\alpha}=\left[x_{\alpha}\right]_{\alpha} \forall \alpha \in I$. But $[x]_{\alpha}=\left[x_{\alpha}\right]_{\alpha} \leftrightarrow x \equiv$ $x_{a}\left(\Phi_{\alpha}\right) \leftrightarrow x+x_{\alpha} \in\left(a_{\alpha}\right) \downarrow\left\{\right.$ where $a+b$ stands for $\left.\left(a \cap b^{\prime}\right) \cup\left(b \cap a^{\prime}\right)\right\}$ or, equivalently, $x+x_{\alpha} \leqq a_{\alpha} \leftrightarrow$ $\left(x+x_{\alpha}\right) a_{\alpha}^{\prime}=0$ (where $a b$ stands for $a \cap b$ ). Hence $\phi$ is surjective if and only if

$$
\bigcup_{\alpha \in I}\left(x+x_{\alpha}\right) a_{\alpha}^{\prime}=0 \leftrightarrow \bigcup_{\alpha \in I}\left(x x_{\alpha}^{\prime} a_{\alpha}^{\prime} \cup x^{\prime} x_{\alpha} a_{\alpha}^{\prime}\right)=0 .
$$

Now, any complete Boolean algebra is infinitely meet (and join) distributive and so $\phi$ is onto $\mathscr{B}^{\infty}$ if and only if the Boolean equation $\left(\bigcup_{\alpha \in I} x_{\alpha}^{\prime} a_{\alpha}^{\prime}\right) x \cup\left(\bigcup_{\alpha \in I} x_{\alpha} a_{\alpha}^{\prime}\right) x^{\prime}=0$ admits a solution. But it is easily seen that a Boolean equation of this form admits a solution if and only if the product of its coefficients is the element 0 and so, by the generalised distributive law $\left(\bigcup_{\alpha \in I} x_{\alpha}\right)\left(\bigcup_{\beta \in J} y_{\beta}\right)=\bigcup_{\alpha \in I, \beta \in J} x_{\alpha} y_{\beta}$, which is known to hold in any complete Boolean algebra, it follows that $\phi$ is onto $\mathscr{B}^{\infty}$ if and only if $\bigcup_{\alpha, \beta \in I} x_{\alpha}^{\prime} a_{\alpha}^{\prime} x_{\beta} a_{\beta}^{\prime}=0$. Once again

$$
\begin{aligned}
\bigcup_{\alpha, \beta \in I} x_{\alpha}^{\prime} a_{\alpha}^{\prime} x_{\beta} a_{\beta}^{\prime}=0 & \leftrightarrow x_{\alpha}^{\prime} x_{\beta} a_{\alpha}^{\prime} a_{\beta}^{\prime}=0 \forall \alpha, \beta \in I \\
& \leftrightarrow x_{\alpha}^{\prime} x_{\beta}\left(a_{\alpha} \cup a_{\beta}\right)^{\prime}=0 \forall \alpha, \beta \in I \\
& \leftrightarrow x_{\alpha}^{\prime} x_{\beta} \leqq a_{\alpha} \cup a_{\beta} \forall \alpha, \beta \in I .
\end{aligned}
$$

On interchanging the roles of $\alpha$ and $\beta$ in the latter condition and combining the two, we have the equivalent condition $x_{\alpha}+x_{\beta} \leqq a_{\alpha} \cup a_{\beta} \forall \alpha, \beta \in I$. But $a_{\alpha} \cup a_{\beta} \in \nabla \forall \alpha, \beta \in I$, so that $a_{\alpha} \cup a_{\beta}=a_{y}$ for some $\gamma \in I$, which is easily seen to coincide with the greatest lower bound $\alpha \wedge \beta$ of the pair $\alpha, \beta$ in $\langle I ; \leqq\rangle$. In summary then, $\phi$ is onto $\mathscr{B}{ }^{\infty}$ if and only if $x_{\alpha}+x_{\beta} \leqq a_{\alpha \wedge \beta}$, which, on writing $\gamma$ for $\alpha \wedge \beta$, is equivalent to $x_{\alpha}+x_{\beta} \in\left(a_{\gamma}\right) \downarrow \forall \alpha, \beta \in I \leftrightarrow x_{\alpha} \equiv x_{\beta}\left(\Phi_{\gamma}\right) \forall \alpha, \beta \in I \leftrightarrow\left[x_{\alpha}\right]_{\gamma}=\left[x_{\beta}\right]_{y}$ $\forall \alpha, \beta \in I$. But $\gamma \leqq \alpha, \beta$ implies $\left[x_{\gamma}\right]_{\gamma}=t(\gamma)=\phi_{y \alpha}(t(\alpha))=\left[x_{\alpha}\right]_{y}$ and similarly $\left[x_{\gamma}\right]_{y}=\left[x_{\beta}\right]_{\gamma}$, so that $\left[x_{\alpha}\right]_{y}=\left[x_{\beta}\right]_{y} \forall \alpha, \beta \in I$. Hence $\phi$ is onto $\mathscr{B}^{\infty}$ and is therefore the required inverse limit representation of $\mathscr{B}$.

\section{REFERENCES}

1. G. Birkhoff, Lattice Theory, Amer. Math. Soc. Colloqium Publications Vol. 25, 3rd edition (Providence, R.I., 1967).

2. G. Grätzer, Universal Algebra (New York, 1968).

3. F. Haimo, Some limits of Boolean algebras. Proc. Amer. Math. Soc. 2 (1951), 566-576.

\section{UNIVERSITY OF GLASGOW}

GLASGOW, G12 8QQ 